\title{
REPRESENTATION OF BILINEAR FORMS IN HILBERT SPACE BY LINEAR OPERATORS $\left(^{1}\right)$
}

\author{
BY \\ ALAN MCINTOSH
}

1. Introduction. This paper is concerned with representing accretive bilinear forms in a Hilbert space by maximal accretive operators in the same space.

The operator $A_{J}$ associated with a bilinear form $J$ is defined to be the operator with largest domain satisfying $J[u, v]=\left(A_{J} u, v\right)$ for all $v \in \mathscr{D}(J)$, the domain of $J$. If $J$ is accretive $(\operatorname{Re} J[u, u] \geqq 0, u \in \mathscr{D}(J))$, then $A_{J}$ is accretive $\left(\operatorname{Re}\left(A_{J} u, u\right) \geqq 0\right.$, $\left.u \in \mathscr{D}\left(A_{J}\right)\right)$. An accretive form $J$ is defined to be representable if $A_{J}$ is maximal accretive (i.e. has no proper accretive extension). This implies that the spectrum of $A_{J}$ is contained in the right half of the complex plane.

Our aim is to give conditions on $J$ under which $J$ is representable.

It is clear that a bounded accretive form on $\mathscr{H}$ is representable (cf. Appendix). The first result of this kind for an unbounded form was derived by Friedrichs (1934) when he proved that the operator associated with a closed semibounded hermitian form is selfadjoint (cf. [2, Chapter 6]). This result was extended to a more general case by $\mathrm{T}$. Kato who showed that a closed regularly accretive form is representable (see $\S 4$ ). (Recall that an accretive form is regularly accretive if $|\operatorname{Im} J[u, u]| \leqq \gamma \operatorname{Re} J[u, u]$ for all $u \in \mathscr{D}(J)$ and some $\gamma \geqq 0$.)

In this paper we consider accretive forms that are not necessarily regularly accretive. A representation theorem for such forms is presented in $\$ 3$. Then in $\S 4$ it is shown that the known result for regularly accretive forms is a corollary of this theorem.

In $\S 5$ we turn to the theory of partial differential equations, and investigate the form

with domain

$$
J[u, v]=\int_{\Omega} \sum f_{j k} \frac{\partial u}{\partial x_{j}} \frac{\partial \bar{v}}{\partial x_{k}}
$$

$$
\left\{u \in L^{2}(\Omega) \mid \exists \frac{\partial u}{\partial x_{j}} \text { for all } j \text {, and } \int\left|\sum f_{j k} \frac{\partial u}{\partial x_{j}} \frac{\partial \bar{u}}{\partial x_{k}}\right|<\infty\right\}
$$

(where $\Omega$ is an open subset of $\boldsymbol{R}^{m}, m \geqq 1$, and $f_{j k}$ are $C^{1}$ functions on $\Omega$ ). Suppose

Received by the editors July 22, 1966.

(1) This work is contained in the author's doctoral dissertation which was written under the supervision of Professor F. Wolf at the University of California, Berkeley. The research was supported in part by a grant from the Air Force Office of Scientific Research, grant AFAFOSR-1071-66. 
that the values of $\sum f_{j k}(x) \xi_{j} \xi_{k}$ lie in a sector $\{z \in C|\operatorname{Re} z>0,| \operatorname{Im} z \mid \leqq \gamma \operatorname{Re} z\}$ of the complex plane $C$, for all $\xi \in C^{m}$ and all $x \in \Omega$. Then $J$ is known to be closed regularly accretive and hence representable (see Theorem 5.2). We prove in Theorem 5.3 that $J$ is representable even when this sector is allowed to rotate about zero as $x$ varies in $\Omega$, so long as $J$ remains accretive, and the rotation satisfies certain conditions. A simple example of a form that is not regularly accretive but which satisfies the hypotheses of this theorem is obtained when $m=1$ and $\Omega=(0, \infty)$ on defining $J$ by

$$
J[u, v]=\int_{0}^{\infty}\left(1+i x^{r} \sin \left(x^{s}\right)\right) \frac{d u}{d x} \frac{d \bar{v}}{d x} d x
$$

with $r>0, s>0$ and $r+s \leqq 1$.

2. Definitions. Throughout this paper, $\mathscr{H}$ denotes a complex Hilbert space, $\boldsymbol{C}$ denotes the complex numbers, and $\boldsymbol{R}$ the reals. Moreover, $\boldsymbol{C}^{l}$ denotes the open left half-plane of $\boldsymbol{C}$, and $\overline{\boldsymbol{C}}^{r}$ denotes the closed right half-plane.

If $\mathscr{D}$ is a dense linear manifold of $\mathscr{H}$, and if $J$ is a map from $\mathscr{D} \times \mathscr{D}$ to $C$ such that

$$
J\left[\alpha_{1} u_{1}+\alpha_{2} u_{2}, v\right]=\alpha_{1} J\left[u_{1}, v\right]+\alpha_{2} J\left[u_{2}, v\right], \quad \alpha_{1}, \alpha_{2} \in C, \quad u_{1}, u_{2}, v \in \mathscr{D} ;
$$

and

$$
J\left[u, \alpha_{1} v_{1}+\alpha_{2} v_{2}\right]=\bar{\alpha}_{1} J\left[u, v_{1}\right]+\bar{\alpha}_{2} J\left[u, v_{2}\right], \quad \alpha_{1}, \alpha_{2} \in C, \quad u, v_{1}, v_{2} \in \mathscr{D} ;
$$

then we say that $J$ is a (bilinear) form in $H$ with domain $\mathscr{D}(J)=\mathscr{D}$.

If $J[u] \stackrel{d}{=} J[u, u] \in \overline{\boldsymbol{C}}^{r}$ for all $u \in \mathscr{D}(J)$, then $J$ is called accretive; if $J[u] \in \boldsymbol{R}$ for all $u \in \mathscr{D}(J)$, then $J$ is called hermitian.

Associated with every form $J$ in $H$ is a linear operator $A_{J}$ defined by

(i) $\mathscr{D}\left(A_{J}\right)=\{u \in \mathscr{D}(J) \mid \exists w \in H \ni J[u, v]=(w, v)$ for all $v \in \mathscr{D}(J)\}$;

(ii) $A_{\mathrm{J}} u=w$.

Then $J[u, v]=\left(A_{J} u, v\right)$ for all $u \in \mathscr{D}\left(A_{J}\right), v \in \mathscr{D}(J)$. Throughout this paper $A_{J}$ denotes the operator defined in this way.

If $J$ is accretive, then $A_{J}$ is accretive (i.e. $\left(A_{J} u, u\right) \in \bar{C}^{r}$ for all $u \in \mathscr{D}\left(A_{J}\right)$ ). However, $\mathscr{D}\left(A_{J}\right)$ need not be dense in $\mathscr{H}$ and could even be just $\{0\}$. Our aim is to show that if $J$ satisfies certain conditions, $A_{J}$ is maximal accretive (i.e. $A_{J}$ is a densely defined accretive operator with no proper accretive extension). Then $A_{J}$ is closed, and has $\boldsymbol{C}^{l}$ in its resolvent set.

When $J$ is an accretive form, and its associated operator $A_{J}$ is maximal accretive, we say that $J$ is representable, and that $J$ is represented by $A_{J}$.

For the theory of accretive operators, see the article by Phillips [4, pp. 199-202] on dissipative operators. (A dissipative operator is the negative of an accretive one.)

When proving that an operator is maximal accretive, we will use the following result of Phillips: 
2.1. Proposition. Let $A$ be an accretive operator in $H$ such that $\mathscr{R}(A-\lambda I)=\mathscr{H}$ for some $\lambda \in C^{l}$. Then $A$ is maximal accretive.

We conclude this section with the following definition:

If $\alpha, \beta \in C, \alpha \neq 0$, then $\alpha J+\beta$ denotes the bilinear form with domain $\mathscr{D}(J)$ defined by

$$
(\alpha J+\beta)[u, v]=\alpha J[u, v]+\beta(u, v) .
$$

Note that $A_{(\alpha J+\beta)}=\alpha A_{J}+\beta I$.

\section{Representation theorem.}

3.1. THEOREM. Let $J$ be an accretive bilinear form with domain $\mathscr{D}(J)$ dense in $\mathscr{H}$. Suppose that $\mathscr{D}(J)$ can be given the structure of a Hilbert space $\mathscr{H}_{1}$ (with inner product $(\cdot, \cdot)_{1}$ and norm $\left.\|\cdot\|_{1}\right)$ satisfying the following properties:

(i) the embedding $j: \mathscr{H}_{1} \rightarrow \mathscr{H}$ is continuous;

(ii) $J$, considered as a form on $\mathscr{H}_{1}$, is bounded;

(iii) if

$$
\sup _{0 \neq w \in \mathscr{H}_{1}} \frac{\left|(J+1)\left[u_{n}, w\right]\right|}{\|w\|_{1}} \rightarrow 0
$$

for a sequence $\left\{u_{n}\right\}$ in $\mathscr{H}_{1}$, then $u_{n} \rightarrow 0$ in $\mathscr{H}_{1}$.

Then $J$ is representable, and $\mathscr{D}\left(A_{J}\right)$ is dense in $\mathscr{H}_{1}$.

REMARK. If (i) and (ii) are satisfied, then (by Theorem A1.2) there exists a continuous linear operator $B$ in $\mathscr{H}_{1}$ such that

$$
(J+1)[u, v]=(B u, v)_{1}, \quad u, v \in \mathscr{H}_{1} .
$$

It will be proved that $B$ is one-to-one. So (iii) can be replaced by the equivalent condition:

(iii) $B^{-1}$ is continuous.

Proof. $B$ has the following properties:

(a) $B$ is one-to-one: Suppose an element $u \in \mathscr{H}_{1}$ satisfies $B u=0$. Then

$$
\begin{aligned}
(J+1)[u] & =(B u, u)_{1}=0 . \\
\therefore u & =0 \quad\left(\text { for if } u \neq 0, \text { then } 0 \leqq \operatorname{Re} J[u]=-\operatorname{Re}\|u\|^{2}<0\right) .
\end{aligned}
$$

(b) $\mathscr{R}(B)$ is dense in $\mathscr{H}_{1}$ : The proof of this fact is similar to the proof of (a).

(c) $\mathscr{R}(B)=\mathscr{H}_{1}$ : This follows from the fact that $B^{-1}$ is closed, continuous and densely defined.

If $j^{*}: \mathscr{H} \rightarrow \mathscr{H}_{1}$ is the adjoint of $j$, it is readily seen that $j^{*}$ is a one-to-one bounded linear operator with $\mathscr{D}\left(j^{*}\right)=\mathscr{H}$ and $\mathscr{R}\left(j^{*}\right)$ dense in $\mathscr{H}_{1}$.

Define the linear operator $A_{1}$ in $\mathscr{H}$ by $A_{1}=\left(j^{*}\right)^{-1} B j^{-1}$. Then $A_{1}$ has range $\mathscr{H}$ 
(because $\mathscr{R}\left(j^{-1}\right)=\mathscr{D}(B), \mathscr{R}(B) \supseteq \mathscr{D}\left(\left(j^{*}\right)^{-1}\right)$, and $\left.\mathscr{R}\left(\left(j^{*}\right)^{-1}\right)=\mathscr{H}\right)$. We now show that $A_{1}=A_{\mathrm{J}}+I$.

$$
\begin{aligned}
\mathscr{D}\left(A_{1}\right) & =\left\{u \in \mathscr{H} \mid \exists w \in \mathscr{H} \ni j^{*} w=B j^{-1} u\right\} \\
& =\left\{u \in \mathscr{H}_{1} \mid \exists w \in \mathscr{H} \ni(w, v)=(B u, v)_{1}, v \in \mathscr{H}_{1}\right\} \\
& =\left\{u \in \mathscr{H}_{1} \mid \exists w \in \mathscr{H} \ni(w, v)=(J+1)[u, v], v \in \mathscr{H}_{1}\right\} \\
& =\mathscr{D}\left(A_{J}\right) ; \text { and } \\
A_{J} u & =w-u=A_{1} u-u .
\end{aligned}
$$

That is, $A_{1}=A_{\mathrm{J}}+I$.

Thus $\mathscr{R}\left(A_{J}+I\right)=\mathscr{H}$, and so, by Proposition 2.1, $A_{J}$ is maximal accretive.

It remains to be shown that $\mathscr{D}\left(A_{J}\right)$ is dense in $\mathscr{H}_{1}$. Now $\mathscr{D}\left(A_{J}\right)\left(=\mathscr{D}\left(A_{1}\right)\right)$, considered as a subset of $\mathscr{H}_{1}$, is really $\mathscr{D}\left(A_{1} j\right)$, and $\mathscr{D}\left(A_{1} j\right)=\mathscr{R}\left(B^{-1} j^{*}\right)$. So we must show that $\mathscr{R}\left(B^{-1} j^{*}\right)$ is dense in $\mathscr{H}_{1}$. This follows from the fact that $\mathscr{R}\left(j^{*}\right)$ is dense in $\mathscr{H}_{1}$, and $B^{-1}$ is a bounded linear operator of $\mathscr{H}_{1}$ onto $\mathscr{H}_{1}$.

REMARK. The statements of various other representation theorems can be found in [3]. Proofs of these results are contained in the author's doctoral dissertation.

4. Regularly accretive forms. A bilinear form $J$ can be decomposed into its real and imaginary parts as follows. Define the forms $J^{*}, J_{1}$ and $J_{2}$ with domains $\mathscr{D}\left(J^{*}\right)=\mathscr{D}\left(J_{1}\right)=\mathscr{D}\left(J_{2}\right)=\mathscr{D}(J)$ by

$$
\begin{aligned}
J^{*}[u, v] & =\overline{J[v, u]}, \\
J_{1} & =\operatorname{Re} J=\frac{1}{2}\left(J+J^{*}\right),
\end{aligned}
$$

and

$$
J_{2}=\operatorname{Im} J=\frac{1}{2 i}\left(J-J^{*}\right)
$$

$J_{1}$ and $J_{2}$ are hermitian forms, called the real part of $J$ and the imaginary part of $J$ respectively. The terminology is natural because $J[u, v]=J_{1}[u, v]+i J_{2}[u, v]$ for $u, v \in \mathscr{D}(J)$, and because $J_{1}[u]=\operatorname{Re}(J[u])$ and $J_{2}[u]=\operatorname{Im}(J[u])$ for $u \in \mathscr{D}(J)$.

Note that $J$ is accretive iff $J_{1}$ is nonnegative $\left(J_{1}[u] \geqq 0, u \in \mathscr{D}(J)\right)$. If, in addition, $J_{2}$ is bounded by $J_{1}\left(\exists \gamma \geqq 0 \ni\left|J_{2}[u]\right| \leqq \gamma J_{1}[u], u \in \mathscr{D}(J)\right)$, then $J$ is called regularly accretive.

$\mathscr{D}(J)$ becomes a pre-Hilbert space $\mathscr{H}_{J}$ with inner product $(\cdot, \cdot)_{J}$ and norm $\|\cdot\|_{J}$ on defining

$$
(u, v)_{J}=(u, v)+J_{1}[u, v]
$$

and

$$
\|u\|_{J}^{2}=(u, u)_{J}=\|u\|^{2}+J_{1}[u] .
$$

4.1. Definition. A regularly accretive form $J$ is closed if $\mathscr{H}_{J}$ is complete. 
4.2. THEOREM. A closed regularly accretive form $J$ is representable, and $\mathscr{D}\left(A_{J}\right)$ is dense in $\mathscr{H}_{\mathrm{J}}$.

Proof. We let the $\mathscr{H}_{1}$ of Theorem 3.1 be $\mathscr{H}_{J}$ and verify conditions (i), (ii) and (iii)', thus obtaining the desired result.

(i) is clearly satisfied, and (ii) follows from the inequality

$$
|J[u]|=\left|J_{1}[u]\right|+\left|J_{2}[u]\right| \leqq(1+\gamma) J_{1}[u] \leqq(1+\gamma)\|u\|_{J}^{2}, \quad u \in \mathscr{D}(J) .
$$

Since $|(J+1)[u]| \geqq\|u\|_{J}^{2}$, (iii)' is a consequence of Theorem A1.3.

This theorem is well known, but it has been included here to show that it is an immediate consequence of Theorem 3.1. The proof we have given is essentially the same as that given by Kato [2, p. 329].

5. Application to partial differential equations. Throughout this section, $\Omega$ denotes an open subset of the $m$-dimensional space $\boldsymbol{R}^{m}, m \geqq 1 ; \mathscr{H}=L^{2}(\Omega)$; and $f_{j k}(j, k=1, \ldots, m)$ are complex-valued $C^{1}$ functions defined on $\Omega$. The real and imaginary parts of the matrix $\left(f_{j k}\right)$ are denoted by $\left(g_{j k}\right)$ and $\left(h_{j k}\right)$ respectively. I.e., $g_{j k}=\frac{1}{2}\left(f_{j k}+\bar{f}_{k j}\right)$, and $h_{j k}=(1 / 2 i)\left(f_{j k}-\bar{f}_{k j}\right) . D_{j}=\partial / \partial x_{j}$ denotes differentiation in the generalized sense. So, if $u$ is a locally integrable function, the statement " $\exists D_{j} u$ " means "the distribution derivative $D_{j} u$ is a (locally integrable) function."

When investigating a second order partial differential operator

$$
\sum \frac{\partial}{\partial x_{k}} f_{j k}(x) \frac{\partial}{\partial x_{j}}
$$

that satisfies $\sum g_{j k}(x) \xi_{j} \xi_{k}>0$ for all $x \in \Omega$ and $0 \neq \xi=\left(\xi_{1}, \ldots, \xi_{m}\right) \in C^{m}$, we can construct the form

$$
J[u, v]=\int_{\Omega} \sum f_{j k} \frac{\partial u}{\partial x_{j}} \frac{\overline{\partial v}}{\partial x_{k}}
$$

with suitable domain, show that $J$ is representable, and thus obtain the corresponding maximal accretive operator $A_{J}$ (cf. [5], [6]). It is known [5] that if the values of $\sum f_{j k}(x) \xi_{j} \xi_{k}$ lie in a sector $\{z \in C|\operatorname{Re} z>0,| \operatorname{Im} z \mid \leqq k \operatorname{Re} z\}$ for all $x \in \Omega$ and $0 \neq \xi \in C^{m}$, and if $\mathscr{D}(J)$ is suitably chosen, then $J$ is representable. This result is proved in Theorem 5.2 (with $\mathscr{D}(J)$ chosen to give the Neumann operator), and then generalized in Theorem 5.3 to the case where the sector is allowed to rotate about zero as $x$ varies in $\Omega$. Some remarks on the Dirichlet operator follow Theorem 5.5.

5.1. Lemma. Suppose that

and

$$
\sum_{j, k=1}^{m} g_{j k}(x) \xi_{j} \xi_{k}>0
$$

$$
\left|\sum_{j, k=1}^{m} h_{j k}(x) \xi_{j} \xi_{k}\right| \leqq \gamma \sum_{j, k=1}^{m} g_{j k}(x) \xi_{j} \xi_{k}
$$


for some $\gamma \geqq 0$, for all $x \in \Omega$, and all $\xi=\left(\xi_{1}, \ldots, \xi_{m}\right) \in C^{m}$ (complex m-dimensional vector space). Then

$$
\begin{aligned}
\sum g_{j k}(x) \xi_{j} \xi_{k} & \leqq\left|\sum f_{j k}(x) \xi_{j} \xi_{k}\right| \leqq(1+\gamma) \sum g_{j k}(x) \xi_{j} \xi_{k} ; \\
\sum g_{j k}(x)\left(\xi_{j}+\eta_{j}\right)\left(\xi_{k}+\bar{\eta}_{k}\right) & \leqq 2 \sum g_{j k}(x) \xi_{j} \xi_{k}+2 \sum g_{j k}(x) \eta_{j} \bar{\eta}_{k} ; \\
\left|\sum f_{j k}(x) \xi_{j} \bar{\eta}_{k}\right|^{2} & \leqq(1+\gamma)^{2}\left|\sum f_{j k}(x) \xi_{j} \xi_{k}\right|\left|\sum f_{j k}(x) \eta_{j} \bar{\eta}_{k}\right|,
\end{aligned}
$$

for all $x \in \Omega ; \xi, \eta \in C^{m}$.

Moreover, if $\Omega^{*} \subset \subset \Omega\left(\Omega^{*}\right.$ is an open precompact subset of $\left.\Omega\right)$, then there exist two positive numbers $\sigma_{1}, \sigma_{2}$ such that

$$
\sigma_{1} \sum\left|\xi_{j}\right|^{2} \leqq \sum g_{j k}(x) \xi_{j} \xi_{k} \leqq \sigma_{2} \sum\left|\xi_{j}\right|^{2},
$$

for all $x \in \Omega^{*}, \xi \in C^{m}$.

Proof. Let $x \in \Omega$. Then $C^{m}$ becomes a Hilbert space $\mathscr{H}_{x}$ on introducing the inner product $(\xi, \eta)_{x}=\sum g_{j k}(x) \xi, \bar{\eta}_{k} . A[\xi, \eta]=\sum h_{j k}(x) \xi_{j} \bar{\eta}_{k}$ is a bounded hermitian form on $\mathscr{H}_{x}$. So (1) and (2) are clear, and (3) is a consequence of Theorem A1.2. (4) follows from the continuity of $g_{j k}$.

5.2. Theorem. Assume that

$$
\sum g_{j k}(x) \xi_{j} \xi_{k}>0 \text { and }\left|\sum h_{j k}(x) \xi_{j} \xi_{k}\right| \leqq \gamma \sum g_{j k}(x) \xi_{j} \xi_{k},
$$

for all $x \in \Omega$, all $\xi \in C^{m}$ and some $\gamma \geqq 0$. Then the bilinear form $K$ in $\mathscr{H}$ defined by

$$
K[u, v]=\int_{\Omega} \sum f_{j k} D_{j} u \overline{D_{k} v}
$$

with domain

$$
\mathscr{D}(K)=\left\{u \in \mathscr{H} \mid \exists D_{j} u \text { for all } j, \text { and } \int_{\Omega}\left|\sum f_{j k} D_{j} u \overline{D_{k} u}\right|<\infty\right\},
$$

is a closed regularly accretive form and thus representable (by Theorem 4.2).

Proof. If $K_{1}=\operatorname{Re} K$ and $K_{2}=\operatorname{Im} K$, then

$$
K_{1}[u]=\int \sum g_{j k} D_{j} u \overline{D_{k} u} \geqq 0
$$

and

$$
\left|K_{2}[u]\right|=\left|\int \sum h_{j k} D_{j} u \overline{D_{k} u}\right| \leqq \int\left|\sum h_{j k} D_{j} u \overline{D_{k} u}\right| \leqq \gamma K_{1}[u],
$$

for all $u \in \mathscr{D}(K)$. Hence $K$ is regularly accretive. Our task is to prove that the preHilbert space $\mathscr{H}_{1}$, constructed from $\mathscr{D}(K)$ by introducing the inner product $(u, v)_{1}=(u, v)+K_{1}[u, v]$, is complete. 
We use the fact that the Sobolev space $W_{1}^{2}(\Omega)$, defined by

$$
W_{1}^{2}(\Omega)=\left\{u \in \mathscr{H} \mid \exists D_{j} u \text { for all } j \text {, and } \int \sum\left|D_{j} u\right|^{2}<\infty\right\},
$$

with inner product

$$
(u, v)_{\Omega}=(u, v)+\int \sum D_{j} u \overline{D_{j} v}
$$

is a Hilbert space.

Let $\left\{u_{n}\right\}$ be a Cauchy sequence in $\mathscr{H}_{1}$. There exists $u \in \mathscr{H}$ such that $u_{n} \rightarrow u$ in $\mathscr{H}$. By (4), $\left\{u_{n} \mid \Omega^{*}\right\}$ is Cauchy in $W_{1}^{2}\left(\Omega^{*}\right)$ for each $\Omega^{*} \subset \subset \Omega$. So $u \mid \Omega^{*} \in W_{1}^{2}\left(\Omega^{*}\right)$ and $u_{n}\left|\Omega^{*} \rightarrow u\right| \Omega^{*}$ in $W_{1}^{2}\left(\Omega^{*}\right)$. In particular, we see that $D_{j} u$ exists for all $j$ (for the existence of $D_{j} u$ is a local property of $u$ ). Furthermore, for each $\Omega^{*}$,

$$
\begin{aligned}
\int_{\Omega^{*}} \sum g_{j k} D_{j} u \overline{D_{k} u} & \leqq C \int_{\Omega^{*}} \sum\left|D_{j} u\right|^{2} \quad \text { (by (4)) } \\
& \leqq 1+C \sup _{n} \int_{\Omega^{*}} \sum\left|D_{j} u_{n}\right|^{2} \\
& \leqq 1+C^{\prime} \sup _{n} \int_{\Omega} \sum g_{j k} D_{j} u_{n} \overline{D_{k} u_{n}} \quad \text { (by (4)) } \\
& \leqq C^{\prime \prime} \quad\left(\because\left\{u_{n}\right\} \text { is a bounded set in } \mathscr{H}_{1}\right) . \\
\therefore \int_{\Omega} \sum g_{j k} D_{j} u \overline{D_{k} u} & \leqq C^{\prime \prime} .
\end{aligned}
$$

So $u \in \mathscr{H}_{1}$. There remains the task of showing that $u_{n} \rightarrow u$ in $\mathscr{H}_{1}$. Let $\varepsilon>0$. Choose $N$ such that $\left\|u_{n}-u_{m}\right\|_{1}^{2}<\varepsilon$ for all $n, m \geqq N$. Choose $\Omega^{*} \subset \subset \Omega$ such that

$$
\int_{\Omega \sim \Omega^{*}}\left|u_{N}\right|^{2}+\sum g_{j k} D_{j} u_{N} \overline{D_{k} u_{N}}<\varepsilon
$$

and

$$
\int_{\Omega \sim \Omega^{*}}|u|^{2}+\sum g_{j k} D_{j} u \overline{D_{k} u}<\varepsilon
$$

Let $n \geqq N$. Then

$$
\begin{aligned}
\int_{\Omega \sim \Omega^{*}}\left|u_{n}\right|^{2}+\sum g_{j k} D_{j} u_{n} \overline{D_{k} u_{n} \leqq} & 2 \int_{\Omega \sim \Omega^{*}}\left|u_{n}-u_{N}\right|^{2}+\sum g_{j k} D_{j}\left(u_{n}-u_{N}\right) \overline{D_{k}\left(u_{n}-u_{N}\right)} \\
& +2 \int_{\Omega \sim \Omega^{*}}\left|u_{N}\right|^{2}+\sum g_{j k} D_{j} u_{N} \overline{D_{k} u_{N}} \quad \text { (by (2)) } \\
< & 4 \varepsilon .
\end{aligned}
$$

By (4), and the fact that $u_{n}\left|\Omega^{*} \rightarrow u\right| \Omega^{*}$ in $W_{1}^{2}\left(\Omega^{*}\right)$, we can choose $M \geqq N$ such that

$$
\int_{\Omega^{*}}\left|u_{n}-u\right|^{2}+\sum g_{j k} D_{j}\left(u_{n}-u\right) \overline{D_{k}\left(u_{n}-u\right)}<\varepsilon, \quad n \geqq M .
$$


$\therefore$, for all $n \geqq M$,

$$
\begin{aligned}
\left\|u_{n}-u\right\|_{1}^{2}= & \int_{\Omega^{*}}+\int_{\Omega \sim \Omega^{*}}\left|u_{n}-u\right|^{2}+\sum g_{j k} D_{j}\left(u_{n}-u\right) \overline{D_{k}\left(u_{n}-u\right)} \\
< & \varepsilon+2 \int_{\Omega \sim \Omega^{*}}\left|u_{n}\right|^{2}+\sum g_{j k} D_{j} u_{n} \overline{D_{k} u_{n}} \\
& +2 \int_{\Omega \sim \Omega^{*}}|u|^{2}+\sum g_{j k} D_{j} u \overline{D_{k} u} \text { (by (2)) } \\
< & \varepsilon+8 \varepsilon+2 \varepsilon=11 \varepsilon .
\end{aligned}
$$

Hence $u_{n} \rightarrow u$ in $\mathscr{H}_{1}$.

The above theorem can be generalized as follows.

5.3. Theorem. Define the bilinear form $J$ in $\mathscr{H}$ by

and

$$
J[u, v]=\int_{\Omega} \exp (i \theta) \sum_{j, k=1}^{m} f_{j k} D_{j} u \overline{D_{k} v}
$$

$$
\mathscr{D}(J)=\left\{u \in H \mid \exists D_{j} u \text { for all } j, \text { and } \int_{\Omega}\left|\sum_{j, k=1}^{m} f_{j k} D_{j} u \overline{D_{k} u}\right|<\infty\right\} .
$$

Suppose that the following properties are satisfied:

(i) $\sum g_{j k}(x) \xi_{j} \xi_{k}>0$, and $\left|\sum h_{j k}(x) \xi_{j} \xi_{k}\right| \leqq \gamma \sum g_{j k}(x) \xi_{j} \xi_{k}$ for all $x \in \Omega$, all $\xi \in C^{m}$, and some $\gamma \geqq 0$;

(ii) $\theta$ is a real-valued $C^{1}$ function on $\Omega$;

(iii) $J$ is accretive;

(iv) $\left|\sum f_{j k} \cdot D_{j} \theta \cdot D_{k} \theta\right|$ is a bounded function on $\Omega$.

Then $J$ is a representable accretive form.

Proof. Since the form $K$ of Theorem 5.2 is closed, $\mathscr{D}(J)(=\mathscr{D}(K))$ becomes a Hilbert space $\mathscr{H}_{1}$ on introducing the inner product

$$
(u, v)_{1}=(u, v)+K_{1}[u, v]=(u, v)+\int \sum g_{j k} D_{j} u \overline{D_{k} v}
$$

So we can prove the theorem by verifying conditions (i), (ii) and (iii) of Theorem 3.1.

(i) is clearly satisfied, while (ii) follows from the inequality

$$
\begin{aligned}
|J[u]| & =\left|\int \exp (i \theta) \sum f_{j k} D_{j} u \overline{D_{k} u}\right| \\
& \leqq \int\left|\sum f_{j k} D_{j} u \overline{D_{k} u}\right| \\
& \leqq(1+\gamma) \int \sum g_{j k} D_{j} u \overline{D_{k} u} \quad(\text { by }(1)) \\
& =(1+\gamma) K_{1}[u] \leqq(1+\gamma)\|u\|_{1}^{2} .
\end{aligned}
$$


To prove (iii), we proceed as follows.

If $u \in \mathscr{H}_{1}$, define $T u$ to be $\exp (i \theta) \cdot u$. Then $D_{j}(T u)=\exp (i \theta)\left(D_{j} u+i u \cdot D_{j} \theta\right)$, $1 \leqq j \leqq m$. We first show that $T u \in \mathscr{H}$ and then that $T u \in \mathscr{H}_{1}$.

(a)

$$
\int|T u|^{2}=\int|u|^{2}<\infty
$$

$\therefore T u \in \mathscr{H}$, and

(5)

$$
\|T u\|=\|u\|
$$

(b) $\int\left|\sum f_{j k} \cdot D_{j}(T u) \cdot \overline{D_{k}(T u)}\right|$

$$
\begin{aligned}
\leqq & \int\left|\sum f_{j k} D_{j} u \overline{D_{k} u}\right|+\int\left|\sum f_{j k} u D_{j} \theta \overline{D_{k} u}\right|+\int\left|\sum f_{j k} D_{j} u D_{k} \theta \cdot \bar{u}\right| \\
& +\int\left|\sum f_{j k} D_{j} \theta D_{k} \theta \cdot u \bar{u}\right| \\
\leqq & \int\left|\sum f_{j k} D_{j} u \overline{D_{k} u}\right|+\left\{\int\left|\sum f_{j k} D_{j} \theta \overline{D_{k} u}\right|^{2}\right\}^{1 / 2}\left\{\int|u|^{2}\right\}^{1 / 2} \\
& +\left\{\int\left|\sum f_{j k} D_{j} u D_{k} \theta\right|^{2}\right\}^{1 / 2}\left\{\int|u|^{2}\right\}^{1 / 2}+C_{1} \int|u|^{2} \quad(\text { by (iv)) } \\
\leqq & \int\left|\sum f_{j k} D_{j} u \overline{D_{k} u}\right|+2(1+\gamma)\|u\|\left\{\int\left|\sum f_{j k} D_{j} \theta D_{k} \theta\right|\left|\sum f_{j k} D_{j} u \overline{D_{k} u}\right|\right\}^{1 / 2} \\
& +C_{1}\|u\|^{2}(\text { by (3)) } \\
\leqq & \int\left|\sum f_{j k} D_{j} u \overline{D_{k} u}\right|+C_{2}\|u\|\left\{\int\left|\sum f_{j k} D_{j} u \overline{D_{k} u}\right|\right\}^{1 / 2}+C_{1}\|u\|^{2} \text { (by (iv)) } \\
< & \infty \quad\left(\because u \in \mathscr{H _ { 1 }}\right) .
\end{aligned}
$$

Hence $T u \in \mathscr{H}_{1}$.

Next we show that $T$ is a bounded operator in $\mathscr{H}_{1}$. Let $u \in \mathscr{H}_{1}$. Then

$$
\begin{aligned}
\|T u\|_{1}^{2}= & \|T u\|^{2}+\int \sum g_{j k} D_{j}(T u) \overline{D_{k}(T u)} \\
\leqq & \|u\|^{2}+\int\left|\sum f_{j k} D_{j}(T u) \overline{D_{k}(T u)}\right| \quad(\text { by }(5) \text { and (1)) } \\
\leqq & \left(1+C_{1}\right)\|u\|^{2}+(1+\gamma) \int \sum g_{j k} D_{j} u \overline{D_{k} u} \\
& +C_{2}(1+\gamma)^{1 / 2}\|u\|\left\{\int \sum g_{j k} D_{j} u \overline{D_{k} u}\right\}^{1 / 2} \quad \text { (by (b) and (1)) } \\
\leqq & C_{3}\left\{\|u\|^{2}+\int \sum g_{j k} D_{j} u \overline{D_{k} u}\right\} \\
= & C_{3}\|u\|_{1}^{2} .
\end{aligned}
$$

So $T$ is bounded. 
Now look at $J[u, T u]$ for $u \in \mathscr{H}_{1}$.

$$
\begin{aligned}
J[u, T u] & \left.=\int \exp (i \theta) \sum f_{j k} D_{j} u \cdot \overline{\exp (i \theta)\left(D_{k} u+i u D_{k} \theta\right.}\right) \\
& =\int \sum f_{j k} D_{j} u\left(\overline{D_{k} u}-i \bar{u} D_{k} \theta\right) .
\end{aligned}
$$

Note that

$$
\begin{aligned}
|Q(u)|^{2} & \leqq \int|u|^{2} \int(1+\gamma)^{2}\left|\sum f_{j k} D_{j} u D_{k} u \|\right| \sum f_{j k} D_{j} \theta D_{k} \theta \mid \quad \text { (by (3)) } \\
& \leqq C\|u\|^{2}|K[u]| \quad \text { (by (iv)). }
\end{aligned}
$$

We are now in a position to verify condition (iii) of Theorem 3.1. Let $\left\{u_{n}\right\}$ be a sequence of nonzero elements of $\mathscr{H}_{1}$ such that

$$
\begin{gathered}
\sup _{0 \neq w \in \mathscr{H}_{1}} \frac{\left|(J+1)\left[u_{n}, w\right]\right|}{\|w\|_{1}} \rightarrow 0 . \\
\therefore \operatorname{Re}(J+1)\left[u_{n}\right] /\left\|u_{n}\right\|_{1} \rightarrow 0 . \\
\therefore\left\|u_{n}\right\|\left\|^{2} /\right\| u_{n} \|_{1} \rightarrow 0 . \\
\therefore\left(u_{n}, T u_{n}\right) /\left\|u_{n}\right\|_{1} \rightarrow 0 \quad \text { (by (5)). }
\end{gathered}
$$

It also follows by (8) that

$$
\begin{aligned}
\left|(J+1)\left[u_{n}, T u_{n}\right]\right| /\left\|T u_{n}\right\|_{1} \rightarrow 0 & \\
\therefore(J+1)\left[u_{n}, T u_{n}\right] /\left\|u_{n}\right\|_{1} \rightarrow 0 & (\because T \text { is bounded). } \\
\therefore J\left[u_{n}, T u_{n}\right] /\left\|u_{n}\right\|_{1} \rightarrow 0 & \text { (by (10)). } \\
\therefore\left(K\left[u_{n}\right]-Q\left(u_{n}\right)\right) /\left\|u_{n}\right\|_{1} \rightarrow 0 & \text { (by (6)). } \\
\therefore\left(\left|K\left[u_{n}\right]\right|-\left|Q\left(u_{n}\right)\right|\right) /\left\|u_{n}\right\|_{1} \rightarrow 0 . & \\
\therefore\left(\left|K\left[u_{n}\right]\right|-\left|Q\left(u_{n}\right)\right|+\frac{1}{4} C\left\|u_{n}\right\|^{2}\right) /\left\|u_{n}\right\|_{1} \rightarrow 0 &
\end{aligned}
$$

(by (9), where $C$ is the constant in equation (7)).

It follows by (7) that the above expression is greater than the nonnegative expression

$$
\frac{\left|K\left[u_{n}\right]\right|-C^{1 / 2}\left|K\left[u_{n}\right]\right|^{1 / 2}\left\|u_{n}\right\|+\frac{1}{4} C\left\|u_{n}\right\|^{2}}{\left\|u_{n}\right\|_{1}} .
$$

So this sequence tends to zero. Hence

$$
\begin{gathered}
\left(\left|K\left[u_{n}\right]\right|^{1 / 2}-\frac{1}{2} C^{1 / 2}\left\|u_{n}\right\|\right) /\left\|u_{n}\right\|_{1}^{1 / 2} \rightarrow 0 . \\
\therefore\left|K\left[u_{n}\right]\right| /\left\|u_{n}\right\|_{1} \rightarrow 0 \quad \text { (by (9)). } \\
\therefore K_{1}\left[u_{n}\right] /\left\|u_{n}\right\|_{1} \rightarrow 0 .
\end{gathered}
$$

So, by (9) and (11), we finally have

$$
\left\|u_{n}\right\|_{1}=\left\|u_{n}\right\|_{1}^{2} /\left\|u_{n}\right\|_{1}=\left(\left\|u_{n}\right\|^{2}+K_{1}\left[u_{n}\right]\right) /\left\|u_{n}\right\|_{1} \rightarrow 0 .
$$

Hence (iii) is satisfied and so the theorem is proved. 
5.4 CoRollary. If $A_{J}$ is the operator associated with $J$, then $\mathscr{D}\left(A_{J}\right)$ is dense in $\mathscr{H}_{1}$.

This is another consequence of the fact that the three conditions of Theorem 3.1 are satisfied.

The operator $A_{J}$ is the Neumann operator (cf. [5]) associated with the formal differential operator $A$ defined by

$$
A u=\sum D_{k}\left\{\exp (i \theta) f_{j k} D_{j} u\right\}
$$

Since $A_{J}$ is maximal accretive, it follows that $C^{l}$ is contained in the resolvent set of $A_{J}$. So we have the existence and uniqueness theorem:

5.5. Theorem. Let $u \in L^{2}(\Omega)$ and $\lambda \in C^{l}$. Then there exists a unique element $v \in \mathscr{D}\left(A_{J}\right)$ satisfying $\left(A_{J}-\lambda\right) v=u$.

We obtain the Dirichlet operator $A_{J_{0}}$ associated with $A$ as follows. Denote the closure of $C_{0}^{\infty}$ in $\mathscr{H}_{1}$ by $\mathscr{H}_{0}$, and let $J_{0}$ denote the restriction of $J$ to $\mathscr{H}_{0}$. By adapting the proof of Theorem 5.3 we see that if conditions (i)-(iv) of that theorem are satisfied (with $J$ replaced by $\left.J_{0}\right)$, then $J_{0}$ is representable, and $\mathscr{D}\left(A_{J_{0}}\right)$ is dense in $\mathscr{H}_{0}$. So Theorem 5.5 remains valid with $A_{J}$ replaced by $A_{J_{0}}$.

Let us return to Theorem 5.3 and see how it generalizes Theorem 5.2. In Theorem 5.2, the values of $\sum f_{j k}(x) \xi_{j} \xi_{k},\left(0 \neq \xi \in C^{m}\right)$ are required to lie in a sector $\{z \in C|\operatorname{Re} z>0,| \operatorname{Im} z \mid \leqq \gamma \operatorname{Re} z\}$ for all $x \in \Omega$. In Theorem 5.3, however, this sector is allowed to rotate about zero as $x$ varies in $\Omega$, so long as $J$ remains accretive and the rotation satisfies certain conditions. In order to get a feeling for these conditions, we will look at the case $m=1$. The results we obtain could be extended to the higher-dimensional cases.

When $m=1$ (and $\Omega$ is connected), Theorem 5.3 becomes:

5.6. THEOREM. Let $(a, b)$ be a (finite or infinite) interval in $R$, and let $\alpha$ be a $C^{1}$ function defined on $(a, b)$ with values in $\bar{C}^{r} \sim\{0\}$. Suppose that $\alpha=\beta \exp (i \theta)$ where $\theta$ is a real-valued $C^{1}$ function on $(a, b), \beta$ is a $C^{1}$ function on $(a, b)$ with values in $a$ sector $\{z \in C|| \operatorname{Im} z \mid \leqq \gamma \operatorname{Re} z\}$ for some $\gamma \geqq 0$, and $|\alpha||d \theta / d x|^{2}$ is bounded on $(a, b)$. Then the form $J$ in $L^{2}(a, b)$ defined by

and

$$
J[u, v]=\int_{a}^{b} \alpha \frac{d u}{d x} \frac{d \bar{v}}{d x}
$$

$$
\mathscr{D}(J)=\left\{u \in L^{2}(a, b) \mid \frac{d u}{d x} \text { exists, and } \int_{a}^{b}|\alpha|\left|\frac{d u}{d x}\right|^{2}<\infty\right\}
$$

is a representable accretive form.

5.7. Corollary. Let $(a, b)$ be an interval in $R$, and let $\alpha$ be a $C^{1}$ function on $(a, b)$ with values in a half plane $\{z \in C \mid \delta \leqq \operatorname{Re} z\}$ for some $\delta>0$. Suppose that there exist points $a^{\prime}$ and $b^{\prime}\left(a<a^{\prime} \leqq b^{\prime}<b\right)$ such that $\operatorname{Im} \alpha$ is semibounded on both $\left(a, a^{\prime}\right)$ and $\left(b^{\prime}, b\right)$. Then the form $J$ defined in Theorem 5.6 is representable. 
Proof. (i) Suppose Im $\alpha$ is bounded from below (from above) on both $\left(a, a^{\prime}\right)$ and $\left(b^{\prime}, b\right)$. Then by the compactness of $\left[a^{\prime}, b^{\prime}\right]$, there exists $\delta_{1}$ such that $\operatorname{Im} \alpha(x)$ $\geqq \delta_{1}\left(\operatorname{Im} \alpha(x) \leqq \delta_{1}\right)$ for all $x \in(a, b)$. The result now follows by Theorem 5.6 with $\theta=\frac{1}{2} \tan ^{-1}\left(\delta / \delta_{1}\right)$ and $\gamma=\tan (\pi / 2-\theta)\left(\theta=-\frac{1}{2} \tan ^{-1}\left(\delta / \delta_{1}\right)\right.$ and $\left.\gamma=\tan (\pi / 2+\theta)\right)$.

(ii) Suppose $\operatorname{Im} \alpha$ is bounded from below on $\left(a, a^{\prime}\right)$, and from above on $\left(b^{\prime}, b\right)$. Let $a^{\prime \prime} \in\left(a, a^{\prime}\right)$. Then there exists $\delta_{1}$ such that $\operatorname{Im} \alpha(x) \geqq \delta_{1}$ for all $x \in\left(a, a^{\prime}\right)$, and $\operatorname{Im} \alpha(x) \leqq \delta_{1}$ for all $x \in\left(a^{\prime \prime}, b\right)$. Let $\theta_{0}=\frac{1}{2} \tan ^{-1}\left(\delta / \delta_{1}\right)$. Define $\theta$ to be a $C^{1}$ function from $(a, b)$ to $\left[-\theta_{0}, \theta_{0}\right]$ such that $\theta(x)=\theta_{0}$ for $x \in\left(a, a^{\prime \prime}\right)$ and $\theta(x)=-\theta_{0}$ for $x \in\left(a^{\prime}, b\right)$. Let $\gamma=\tan \left(\pi / 2-\theta_{0}\right)$. Then the hypothesis of Theorem 5.6 is satisfied and so $J$ is representable.

(iii) The proof is similar in the case when $\operatorname{Im} \alpha$ is bounded from above on $\left(a, a^{\prime}\right)$ and from below on $\left(b^{\prime}, b\right)$.

The following proposition is useful when determining whether the conditions of Theorem 5.6 are satisfied.

5.8. Proposition. Let $\alpha$ be a $C^{1}$ function on $(a, b)$ with values in $\bar{C}^{r} \sim\{0\}$. Let $\alpha_{1}=\operatorname{Re} \alpha, \alpha_{2}=\operatorname{Im} \alpha$ and $\theta=\arg \alpha$. Then

$$
|\alpha||d \theta / d x|^{2} \leqq\left|\alpha_{1}\left(d \alpha_{2} / d x\right)-\alpha_{2}\left(d \alpha_{1} / d x\right)\right|^{2}|\alpha|^{-3},
$$

with equality if $\alpha_{1} \neq 0$.

Proof. Let $S=\left\{x \in(a, b) \mid \alpha_{1}(x)>0\right\}$.

(i) Suppose $x \in S$. Then $\theta(x)=\tan ^{-1}\left(\alpha_{2}(x) \cdot \alpha_{1}^{-1}(x)\right)$.

$$
\therefore \frac{d \theta}{d x}=\frac{\alpha_{1}\left(d \alpha_{2} / d x\right)-\alpha_{2}\left(d \alpha_{1} / d x\right)}{\alpha_{1}^{2}} \cdot \frac{\alpha_{1}^{2}}{\alpha_{1}^{2}+\alpha_{2}^{2}} \text {. }
$$

$$
\therefore|\alpha||d \theta / d x|^{2}=\left|\alpha_{1}\left(d \alpha_{2} / d x\right)-\alpha_{2}\left(d \alpha_{1} / d x\right)\right|^{2}|\alpha|^{-3} \text {. }
$$

(ii) Suppose $x \in S^{-}$(the closure of $S$ in $(a, b)$ ). Then, by continuity, (13) still holds.

(iii) Suppose $x \in(a, b) \sim S^{-}$. Then $\theta$ is constant in a neighborhood of $x$, so the left-hand side of (12) equals zero.

We conclude this discussion by giving three simple examples of representable accretive forms. Note that none of these forms is regularly accretive.

(i) $J[u, v]=\int_{-\infty}^{\infty}\left(1+i x^{3}\right) \frac{d u}{d x}(x) \overline{\frac{d v}{d x}(x)} d x$ (in the Hilbert space $L^{2}(-\infty, \infty)$ );

(ii) $J[u, v]=\int_{0}^{\infty}\left(1+i x^{r}-i x^{-s}\right) \frac{d u}{d x}(x) \frac{\overline{d v}(x)}{d x} d x, \quad r, s>0$

(in the Hilbert space $L^{2}(0, \infty)$ );

(iii) $J[u, v]=\int_{1}^{\infty}\left(1+i x^{r} \sin \left(x^{s}\right)\right) \frac{d u}{d x}(x) \frac{\overline{d v}(x)}{d x} d x, \quad 0<r, \quad 0<s$, $r+s \leqq 1 \quad$ (in the Hilbert space $L^{2}(1, \infty)$ ).

[In each case, $\mathscr{D}(J)$ is defined as in Theorem 5.6.] 
The fact that the first two forms are representable is a direct consequence of Corollary 5.7. To see that the third form is representable, we proceed as follows. Let $\alpha=1+i x^{r} \sin \left(x^{s}\right)$ and let $\theta=\arg \alpha$. Then, by Proposition 5.8,

$$
\begin{aligned}
& |\alpha||d \theta / d x|^{2}=\left|r x^{r-1} \sin \left(x^{s}\right)+s x^{r+s-1} \cos \left(x^{s}\right)\right|^{2} \cdot\left|1+x^{2 r} \sin ^{2}\left(x^{s}\right)\right|^{-3 / 2} \\
& \leqq C \cdot x^{2(r+s-1)} \quad \text { (where } C \text { is a constant) } \\
& \leqq C \quad(\because x \geqq 1 \text { and } r+s \leqq 1) \text {. }
\end{aligned}
$$

The result now follows by applying Theorem 5.6 with $\beta=|\alpha|$ and $\gamma=0$.

\section{Appendix. Bounded forms.}

A1.1. Definition. $J$ is a bounded bilinear form on $\mathscr{H}$ if $\mathscr{D}(J)=\mathscr{H}$ and there exists $\alpha \geqq 0$ such that $|J[u]| \leqq \alpha\|u\|^{2}$ for all $u \in \mathscr{H}$. The smallest such $\alpha$ is called the bound of $J$.

A1.2. THEOREM. The following statements about the form $J$ with domain $\mathscr{H}$ are equivalent:

(i) $J$ is a bounded form on $\mathscr{H}$;

(ii) $\exists \beta \geqq 0$ such that $|J[u, v]| \leqq \beta\|u\| \cdot\|v\|$ for all $u, v \in \mathscr{H}$;

(iii) there exists a bounded linear operator $A_{J}$ in $\mathscr{H}$ such that $J[u, v]=\left(A_{J} u, v\right)$ for all $u, v \in \mathscr{H}$.

Proof. The proof is fairly straightforward, the only difficult part being (i) $\Rightarrow$ (ii). This is proved in Halmos [1, p. 33], where it is shown that if $\alpha$ is the bound of $J$, then $\beta$ can be taken equal to $\alpha$ or $2 \alpha$, depending on whether $J$ is hermitian or not.

A1.3. THEOREM. Let $J$ be a bounded form on $\mathscr{H}$ satisfying $|J[u]| \geqq \delta\|u\|^{2}$ for some $\delta>0$. Then, if $A_{J}$ is the operator defined in Theorem A1.2, $A_{J}$ is 1-1 and its inverse is continuous.

\section{REFERENCES}

1. P. R. Halmos, Introduction to Hilbert space, Chelsea, New York, 1951.

2. T. Kato, Perturbation theory for linear operators, Springer, Berlin, 1966.

3. A. McIntosh, Representation of bilinear forms in Hilbert space by linear operators. I, II, Notices Amer. Math. Soc. 13 (1966), 722, 857.

4. R. S. Phillips, Dissipative operators and hyperbolic systems of partial differential equations, Trans. Amer. Math. Soc. 90 (1959), 193-254.

5. E. T. Poulsen, On the local origin of the essential spectra of elliptic differential operators, J. Math. Mech. 11 (1962), 725-747.

6. F. Wolf, On singular partial differential boundary problems, Ann. Mat. Pura Appl. (IV) 49 (1960), 167-180.

INSTITUTE FOR ADVANCED STUDY, Princeton, New Jersey 